\title{
Photoproduction of upsilon states in ultraperipheral collisions at the CERN Large Hadron Collider within the color dipole approach
}

\author{
M. B. Gay Ducati, F. Kopp, M. V. T. Machado, and S. Martins \\ High Energy Physics Phenomenology Group, GFPAE IF-UFRGS Caixa Postal 15051, CEP 91501-970 \\ Porto Alegre, Rio Grande do Sul, Brazil
}

(Received 19 October 2016; published 18 November 2016)

\begin{abstract}
The exclusive photoproduction of upsilon state $\Upsilon(1 S)$ and its radially excited states $\Upsilon(2 S, 3 S)$ is investigated in the context of ultraperipheral collisions at the LHC energies. Predictions are presented for their production in proton-proton, proton-nucleus, and nucleus-nucleus collision at the energies available at the LHC Run 2. The rapidity and transverse-momentum distributions are shown, and the robustness of the model is tested against the experimental results considering $\psi(1 S, 2 S)$ and $\Upsilon(1 S)$ states. The theoretical framework considered in the analysis is the light-cone color dipole formalism, which includes consistently parton saturation effects and nuclear shadowing corrections.
\end{abstract}

DOI: 10.1103/PhysRevD.94.094023

\section{INTRODUCTION}

The exclusive photoproduction of heavy vector mesons is a kind of diffractive process in which, besides a soft scale characterized by the hadron size, there is clearly a hard scale (mesons mass $m_{V}$ ) that allows one to analyze the reaction from the perturbative QCD point of view. This advantage creates ways to investigate the pomeron exchange, which could lead to a better understanding of this object in terms of QCD. Another advantage in studying this process occurs in ultraperipheral collisions [1], where the impact parameter is larger than the sum of the radius of the interacting hadrons. In this case, the exclusive photoproduction dominates the process through the emission of virtual photons which interact with the target featuring the photon-target cross section. The photon-target interaction amplitude, when considering the light-cone dipole formalism [2], can be written as a convolution between the photon-meson wave functions overlap and the elementary dipole-target cross section [3]. The exclusive quarkonium photoproduction has been investigated both experimentally and theoretically in recent years. It allows one to test perturbative QCD (pQCD) as the masses of these heavy mesons give a perturbative scale for the problem even in the photoproduction limit. An important feature of these exclusive processes at the high-energy regime is the possibility of investigating the hard pQCD Pomeron exchange. At the present energy regime at the LHC, photons can be considered as color dipoles in the mixed light-cone representation, where their transverse size can be considered frozen during the interaction [2]. The corresponding scattering process is characterized by the color dipole cross section describing the interaction of those color dipoles with the nucleon or nucleus target. The referred approach is quite intuitive and brings information on the dynamics beyond the leading logarithmic QCD approach. The dynamics related to the meson formation is given by their wave functions, and to compute predictions for their excited states is a reasonable task [3].

In this work, we investigate the exclusive production of $\Upsilon(1 S)$ and its radially excited states $\Upsilon(2 S)$ and $\Upsilon(3 S)$ in proton-proton, proton-nucleus, and nucleus-nucleus collisions in the LHC energy interval. In previous works [4,5], some of us have considered coherent photoproduction of $J / \psi$ and $\psi(2 S)$ states at various energies in $p p$ and $\mathrm{PbPb}$ collisions at the LHC. Those calculations were carried out in the theoretical framework of the color light-cone dipole formalism [2]. Moreover, in Refs. [6,7], a Regge-pole model that successfully describes the DESY-HERA data was considered for vector meson production, and the correlation between the meson production cross sections in photon-induced reactions at HERA and those in ultraperipheral collisions at the LHC was analyzed. It was shown that the corresponding predictions describe nicely the experimental results from ALICE [8,9] and LHCb $[10,11]$ collaborations on the charmonia production. In the dipole framework, the heavy quark-antiquark fluctuation of the incoming quasireal photons interacts with the target via the dipole cross section, and the result is projected into the wave function of the observed meson state. The photoproduction also gives us ways to investigate the transition between the linear dynamics, governed by DGLAP (Dokshitzer-Gribov-Lipatov-Altarelli-Parisi) and BFKL (Balitskii-Fadin-Kuraev-Lipatov) evolution equations, and the nonlinear dynamics in which the physical process of the partonic recombination, e.g., $g g \rightarrow g$, becomes important. In the energies available at the LHC, the transition of the regime described by the linear dynamics of the emissions chain to a new regime, where the physical process of the recombination of partons becomes important, is expected. It is characterized by the limitation on the maximum phase-space parton density that can be reached in the hadron wave function, the so-called parton saturation phenomenon $[12,13]$. The transition is set by the saturation 
scale $Q_{\text {sat }}$, which is enhanced in the nuclear case. Therefore, the color dipole approach will include both the parton saturation effects in photon-proton interaction as nuclear shadowing effects in the photon-nucleus process. For examples of the values of Bjorken $x$ reached at the LHC (at midrapidity), one has $x \simeq 10^{-4}$ for $p p, x \simeq 10^{-3}$ for $p A$, and $x \simeq 2 \times 10^{-3}$ for $A A$ collisions. Those values are even smaller for very forward rapidities; e.g., for $y=4$ the $x$ value diminishes by a factor 100 .

The paper is organized as follows. In the next section, we summarize the main theoretical information to compute the rapidity and transverse-momentum distributions of $\Upsilon(1 S, 2 S, 3 S)$ states in $p p, p A$, and $A A$ collisions for present and future runs at the LHC. We directly compare the results to the experimental data measured by the $\mathrm{LHCb}$ Collaboration [14] for $\Upsilon(1 S)$ in $p p$ collisions. In the Sec. III, we present the numerical calculations and discuss the main theoretical uncertainties, and a comparison with other approaches is done. In particular, we contrast the main results against the predictions available using the STARlight Monte Carlo [15-17]. In the last section, we show the main conclusions.

\section{THEORETICAL FRAMEWORK}

At high energies, the total cross section for photoproduction of a vector meson $V$ in ultraperipheral $p p$, $p A$, or $A A$ collisions can be written in a factorized way as $[1,16]$

$$
\sigma_{V}=\int d \omega \frac{d N_{\gamma}}{d \omega} \sigma_{\gamma}^{p(A)}(\omega)
$$

where the first term, $d N(\omega) / d \omega$, represents the virtual photons flux and can be calculated from the WeizsäckerWilliams method [1]. It characterizes the photon energy $(\omega)$ distribution emitted by the hadrons. Moreover, the photoproduction cross section, $\sigma_{\gamma}^{p(A)}(\omega)$, quantifies the cross section for the $\gamma+p(A) \rightarrow p(A)+V$ process.

For highly energetic protons as those produced by the LHC beams, the photon flux can be approximated by $[1,16]$

$$
\begin{aligned}
\frac{d N_{\gamma}^{p}}{d \omega} & =\frac{\alpha_{e m}}{2 \pi \omega}\left[1+\left(1-\frac{2 \omega}{\sqrt{s}}\right)^{2}\right] \\
& \times\left[\ln \Omega-\frac{11}{6}+\frac{3}{\Omega}-\frac{3}{2 \Omega^{2}}+\frac{1}{3 \Omega^{3}}\right],
\end{aligned}
$$

where $\Omega=1+0.71 \mathrm{GeV}^{2} / Q_{\min }^{2}$ e $Q_{\min }^{2}=\left(\omega / \gamma_{L}\right)^{2}$, with $\gamma_{L}=\sqrt{s} /\left(2 m_{p}\right)$. On the other hand, for a nucleus having charge $Z$, the photon flux is approximately given by $[1,16]$

$\frac{d N_{\gamma}^{A}}{d \omega}=\frac{2 Z^{2} \alpha_{e m}}{\pi}\left[\xi K_{0}(\xi) K_{1}(\xi)-\frac{\xi^{2}}{2}\left(K_{1}^{2}(\xi)-K_{0}^{2}(\xi)\right)\right]$, where $\xi=\omega\left(R_{p}+R_{A}\right) / \gamma_{L}$ for $p A$ collisions and $\xi=$ $\omega\left(2 R_{A}\right) / \gamma_{L}$ for $A A$ collisions.

In the present work, we consider the color dipole approach for modeling the photon-target interaction. Therefore, the cross section $\sigma_{\gamma}^{p(A)}$ appearing in Eq. (1) will be computed from the following scattering amplitude in the photoproduction limit $\left(Q^{2}=0\right)$ [3],

$A^{\gamma^{*} p(A) \rightarrow V p(A)}(x, \Delta)=\int d^{2} \mathbf{r} \int_{0}^{1} \frac{d z}{4 \pi} \rho_{V}(z, r) A_{q \bar{q}}(x, r, \Delta)$,

where the quantity $\rho_{V}=\left(\Psi_{V}^{*} \Psi\right)_{T}$ represents the overlap of the photon-meson wave functions and $A_{q \bar{q}}$ is the dipoletarget scattering amplitude (assumed to be imaginary). Accordingly, the usual kinematical variables are as follows. $x$ is the Bjorken variable, and the squared momentum transfer in hadron vertex is $t=-\Delta^{2}$. The variables $z$ and $r$ are the longitudinal momentum fraction carried by the quark, and $r$ is the transverse color dipole size.

From Eq. (5), we will consider the simplification that the $\Delta$ dependence on the amplitude is exponential, $A_{q \bar{q}} \propto e^{-B_{V} \Delta^{2} / 2}$. Moreover, one includes the needed corrections related to the real part of amplitude, $\beta=$ $\operatorname{Re} A / \operatorname{Im} A$, and off-diagonal momenta of exchanged gluons, $R_{g}$. Both quantities depend on the effective energy power behavior, $\lambda_{\text {eff }}$, of the scattering amplitude. Thus, the cross section for the exclusive photoproduction for $p p$ collisions reads as $[4,5]$

$\sigma(\gamma p \rightarrow V p)(\omega)=\frac{R_{g}^{2}}{16 \pi B_{V}}\left|A_{p}(x, \Delta=0)\right|^{2}\left(1+\beta^{2}\right)$.

In the above equation, $B_{V}$ is the slope parameter, which characterizes the size of the interaction region. Along the calculation performed here, the energy dependence from the Regge phenomenology [18] was considered to describe the slope parameter,

$$
B_{V}\left(W_{\gamma p}\right)=b_{e l}^{V}+2 \alpha^{\prime} \log \left(\frac{W_{\gamma p}^{2}}{W_{0}^{2}}\right),
$$

with $\quad \alpha^{\prime}=0.164 \mathrm{GeV}^{-2}, \quad W_{0}=95 \mathrm{GeV}, \quad b_{e l}^{\Upsilon_{(1 S)}}=$ $3.68 \mathrm{GeV}^{-2}, b_{e l}^{\Upsilon_{(2 S)}}=3.61 \mathrm{GeV}^{-2}$, and $b_{e l}^{\Upsilon_{(3 S)}}=3.57 \mathrm{GeV}^{-2}$.

In the case of nuclear targets, the coherent photonuclear cross section will be calculated using the expression $[15,16]$

$$
\begin{aligned}
\sigma(\gamma A \rightarrow V A)(\omega)= & R_{g}^{2} \frac{\left|A_{\mathrm{nuc}}(x, \Delta=0)\right|^{2}}{16 \pi}\left(1+\beta^{2}\right) \\
& \times \int_{t_{\min }}^{\infty}|F(t)|^{2} d t,
\end{aligned}
$$


where $t_{\min }=\left(m_{V}^{2} / 2 \omega\right)^{2}$ and the nuclear form factor is given by

$F(q)=\frac{4 \pi \rho_{0}}{A q^{3}}\left[\sin \left(q R_{A}\right)-q R_{A} \cos \left(q R_{A}\right)\right]\left[\frac{1}{1+a^{2} q^{2}}\right]$,

where $q=\sqrt{|t|}$, with $\rho_{0}=0.16 \mathrm{fm}^{-3}$ and $a=$ $0.7 \mathrm{fm} \mathrm{[16].}$

Setting the vector meson wave function [appearing in Eq. (5)] as a quark-antiquark state having spin and polarization structure similar to the photon, the corresponding overlap photon-meson wave function can be written as [19]

$$
\begin{aligned}
\rho_{V}(r, z)= & \hat{e}_{f} e \frac{N_{c}}{\pi z(1-z)}\left\{m_{f}^{2} K_{0}(\varepsilon r) \phi_{T}(r, z)\right. \\
& \left.-\left[z^{2}+(1-z)^{2}\right] \varepsilon K_{1}(\varepsilon r) \partial_{r} \phi_{T}(r, z)\right\},
\end{aligned}
$$

where the effective charge $\hat{\mathrm{e}}_{f}=-1 / 3$ for the $\Upsilon$ states. In contrast to the photon wave functions which can be completely computed from perturbation theory [2], the meson wave function carries a phenomenological input embedded in functions $\phi_{T, L}$. In our calculations, we use the boosted-Gaussian model [19] because it can be applied in a systematic way for excited states. The corresponding function is given by [20]

$$
\phi_{n S}(r, z)=\left[\sum_{k=0}^{n-1} \alpha_{n S, k} R_{n S}^{2} \hat{D}^{2 k}(r, z)\right] G_{n S}(r, z),
$$

with $\alpha_{n S, 0}=1$. The operator $\hat{D}^{2}(r, z)$ is defined by

$$
\hat{D}^{2}(r, z)=\frac{m_{f}^{2}-\nabla_{r}^{2}}{4 z(1-z)}-m_{f}^{2},
$$

where $\nabla_{r}^{2}=\frac{1}{r} \partial_{r}+\partial_{r}^{2}$, and acts on the Gaussian function

$$
\begin{aligned}
G_{n S}(r, z)= & \mathcal{N}_{n S} z(1-z) \exp \left(-\frac{m_{f}^{2} \mathcal{R}_{n S}^{2}}{8 z(1-z)}\right. \\
& \left.-\frac{2 z(1-z) r^{2}}{\mathcal{R}_{n S}^{2}}+\frac{m_{f}^{2} \mathcal{R}_{n S}^{2}}{2}\right) .
\end{aligned}
$$

The parameters $\mathcal{R}_{n S}^{2}$ and $\mathcal{N}_{n S}$ are determined by the normalization conditions and by the decay widths into dileptons [20]. In addition, the parameters $\alpha_{n S, k}$ (for $k>0$ ) are fixed by requiring the wave functions of different states to be orthogonal to each other. For the boosted Gaussian, these parameters were calculated in Ref. [20], and they are summarized in Table I. We call attention to the fact that the remaining literature, in general, uses only information on the $\Upsilon(1 S)$ state and constrains the excited-state cross section using the measured values available at lower energies as provided by the DESY-HERA experiment.

Another important component in Eq. (5) is the dipole scattering amplitude. We use the following
TABLE I. Parameters for a boosted-Gaussian wave function for $\Upsilon(1 S), \Upsilon(2 S)$, and $\Upsilon(3 S)$ states.

\begin{tabular}{lcccccc}
\hline \hline Meson & $m_{f}(\mathrm{GeV})$ & $M_{V}(\mathrm{GeV})$ & $\mathcal{N}_{T}$ & $\mathcal{R}_{n S}^{2}\left(\mathrm{GeV}^{-2}\right)$ & $\alpha_{1 S}$ & $\alpha_{2 S}$ \\
\hline$\Upsilon(1 S)$ & 4.2 & 9.46 & 0.481 & 0.567 & $\ldots$ & $\ldots$ \\
$\Upsilon(2 S)$ & 4.2 & 10.023 & 0.624 & 0.831 & -0.555 & $\ldots$ \\
$\Upsilon(3 S)$ & 4.2 & 10.355 & 0.668 & 1.028 & -1.219 & 0.217 \\
\hline \hline
\end{tabular}

phenomenological models in our analyses: Golec-Biernat and Wusthoff (GBW) [21], Golec-Biernat and WusthoffKozlov, Shoshi and Xian (GBW-KSX) [22], Color Glass Condensate model (CGC) [23], and impact parameter Color Glass Condensate model (BCGC) [24]. The dipole amplitude is related to the color dipole cross section in the form

$$
\sigma_{q \bar{q}}(x, r)=2 \int d^{2} b A_{q \bar{q}}(x, r, b),
$$

bearing in mind that $b$ and $\Delta$ are Fourier conjugates variables.

The GBW model is defined by the ansatz

$$
\sigma_{q \bar{q}}^{\mathrm{GBW}}(x, r)=\sigma_{0}\left(1-e^{-r^{2} Q_{s}^{2}(x) / 4}\right),
$$

where $\sigma_{0}=2 \pi R^{2}$ is a constant and $Q_{s}^{2}(x)=\left(x_{0} / x\right)^{\lambda} \mathrm{GeV}^{2}$ denotes the saturation scale, where the partonic recombination effects become important. A further correction has been introduced in the above model, called the GBW-KSX model [22], including gluon number fluctuation effects. We also consider the CGC model [23], based in the color glass condensate framework, in which gluon saturation effects are incorporated via an approximate solution of the BalitskyKovchegov equation [25-27]. In this trend, two cases were considered: CGC-old parametrization [28], which considers the previous DESY-HERA data, and CGC-new parametrization [29], which considers more recent data from ZEUS and $\mathrm{H} 1$ combined results for inclusive deep inelastic scattering. All the parameters associated to the referred models for the dipole amplitude are shown in Table II. The expression for the CGC model is given by

$$
\sigma_{q \bar{q}}^{\mathrm{CGC}}(x, r)=\sigma_{0}\left\{\begin{array}{ll}
\mathcal{N}_{0}\left(\frac{r Q_{s}}{2}\right)^{\gamma_{\text {eff }}(x, r)}: & r Q_{s} \leq 2 \\
1-\mathrm{e}^{-A \ln ^{2}\left(B r Q_{s}\right)}: & r Q_{s}>2
\end{array},\right.
$$

TABLE II. Parameters and charm quark mass associated to the distinct model for the dipole amplitude (see the text). For the bottom mass, one considers the fixed value $m_{b}=4.5 \mathrm{GeV}$.

\begin{tabular}{lcccc}
\hline \hline & $\sigma_{0}(\mathrm{mb})$ & $x_{0}\left(10^{-4}\right)$ & \multicolumn{1}{c}{$\lambda$} & $\gamma_{s}$ \\
\hline GBW & 29.12 & 0.41 & 0.277 & $\ldots$ \\
GBW $^{k s x}$ & 31.85 & 0.0546 & 0.225 & $\ldots$ \\
CGC $_{\text {old }}$ & 27.33 & 0.1632 & 0.2197 & 0.7376 \\
CGC $_{\text {new }}$ & 21.85 & 0.6266 & 0.2319 & 0.762 \\
BCGC $_{\text {old }}$ & $\cdots$ & 0.0184 & 0.119 & 0.46 \\
\hline \hline
\end{tabular}


where $\gamma_{\text {eff }}(x, r)=2\left(\gamma_{s}+(1 / \kappa \lambda Y) \ln \left(2 / r Q_{s}\right)\right)$ is the effective anomalous dimension, $Y=\ln (1 / x)$, and one has the constant $\kappa=9.9$. The saturation scale takes the same form as in the GBW model. Finally, we have tested also the impact parameter CGC model (named the b-CGC model). The expression for the $\mathrm{b}-\mathrm{CGC}$ model is given by

$\sigma_{q \bar{q}}^{\mathrm{BCGC}}(x, r)=2 \int d^{2} b\left\{\begin{array}{ll}\mathcal{N}_{0}\left(\frac{r Q_{s}}{2}\right)^{\gamma_{\text {eff }}(x, r)}: & r Q_{s} \leq 2 \\ 1-\mathrm{e}^{-A \ln ^{2}\left(B r Q_{s}\right)}: & r Q_{s}>2\end{array}\right.$,

where the parameter $Q_{s}$ depends on the impact parameter,

$$
Q_{s} \equiv Q_{s}(x, b)=\left(\frac{x_{0}}{x}\right)^{\frac{\lambda}{2}}\left[\exp \left(-\frac{b^{2}}{2 B_{\mathrm{CGC}}}\right)\right]^{\frac{1}{2 \gamma_{s}}},
$$

where $B_{\mathrm{CGC}}=7.5 \mathrm{GeV}^{-2}$.

In the next section, we provide predictions to the future (current) runs of LHC for the rapidity and transversemomentum distributions of the $\Upsilon(1 S, 2 S, 3 S)$ states in ultraperipheral collisions.

\section{RESULTS AND DISCUSSIONS}

Let us start the analysis by computing the theoretical predictions for the proton-proton case. In $p p$ collisions, the rapidity distribution of vector meson $V$ is given by

$$
\begin{aligned}
\frac{d \sigma}{d y}(p+p \rightarrow p+p+V)= & S_{\text {gap }}^{2}\left[\omega \frac{d N_{\gamma}^{p}(\omega)}{d \omega} \sigma(\gamma p \rightarrow V+p)\right. \\
& +(y \rightarrow-y)]
\end{aligned}
$$

where the rapidity of the produced meson is related to the photon energy by $y \simeq \ln \left(2 \omega / m_{V}\right)$. The parameter $S_{\text {gap }}^{2}$ quantifies the absorptive corrections [30], and in such a process, one has the presence of a large rapidity gap between the produced meson and the final-state protons. In the present calculation, we will take $S_{\text {gap }}^{2} \approx 0.8-0.9$. Finally, the notation $(y \rightarrow-y)$ indicates the symmetry target projectile in the $p p$ collision. Figure 1 presents the results for photoproduction $\Upsilon(1 S)$ in $p p$ collisions considering the different models presented in the last section. The relative normalization and the overall behavior on rapidity are fairly reproduced by all the models in the forward region in comparison to the experimental results from LHCb Collaboration [14]. Given the present level of the experimental uncertainties, it is not possible to make definitive statements about the precision of the distinct models investigated. The theoretical uncertainty reaches a factor 2 considering the same wave function and distinct dipole cross sections. On the other hand, when the integrated cross sections for $\Upsilon(1 S)$ and $\Upsilon(2 S)$ are considered, a better agreement is achieved in the frontal region $2<\eta<4.5$ by the old versions of the GBW and CGC

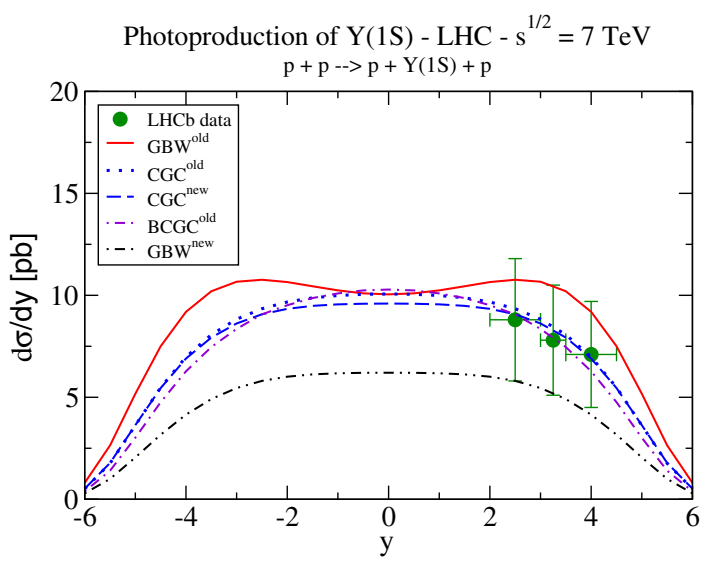

FIG. 1. Rapidity distribution for the $\Upsilon(1 S)$ state in $p p$ collisions at $\sqrt{s}=7 \mathrm{TeV}$. Data are from the LHCb Collaboration [14].

models. The corresponding results for the models and the $\mathrm{LHCb}$ data are shown in Table III. For the sake of completeness, the production of $\psi(1 S)$ and its excited state $\psi(2 S)[10,11]$ have been added (including also the experimental result [14]), corrected for acceptance.

The calculation discussed above can be directly compared to other theoretical approaches. For instance, the present result is consistent with the next-to-leading-order (NLO) pQCD analysis done in Ref. [31], which also compares the outcoming predictions to the $\mathrm{LHCb}$ data. It is verified that the theoretical uncertainty from the color dipole approach is somewhat smaller than that presented there [particularly, leading-order and NLO pQCD predictions present large disparities]. Moreover, it is worth it to note that the present work updates the analysis done in Ref. [20], where the photoproduction of $\Upsilon$ states in $p p$ collisions was done for the first time in the scope of the color dipole approach, also used in Ref. [32] for $\Upsilon(1 S)$. Our results are also similar to the Regge-pole model treatment of vector meson photoproduction as predicted and discussed in Refs. [6,7]. The focus here is to provide predictions for the next (and current) runs of the LHC.

In Fig. 2, predictions are done for the LHC Run 2 at $\sqrt{s}=13 \mathrm{TeV}$ in the $p p$ mode (the notation is the same as the previous plot). The general trend follows the one

TABLE III. Integrated cross sections (in units of $p b$ ) for photoproduction of the $\psi(1 S, 2 S)$ (corrected for acceptance) and $\Upsilon(1 S, 2 S, 3 S)$ states in $p p$ collisions at $\sqrt{s}=7 \mathrm{TeV}$ compared to the $\mathrm{LHCb}$ data $[10,11,14]$ (errors are summed into quadrature).

\begin{tabular}{lrrrccc}
\hline \hline & GBW & CGC $^{\text {old }}$ & $\mathrm{CGC}^{\text {new }}$ & BCGC $^{\text {old }}$ & $\mathrm{GBW}^{k s x}$ & $\mathrm{LHCb}$ \\
\hline$\psi(1 s)$ & 277.60 & 213.69 & 199.58 & 154.57 & 170.81 & $291 \pm 20.24$ \\
$\psi(2 s)$ & 8.40 & 5.94 & 5.98 & 4.13 & 4.39 & $6.5 \pm 0.98$ \\
$\Upsilon(1 s)$ & 25.05 & 20.45 & 20.02 & 19.12 & 12.5 & $9.0 \pm 2.7$ \\
$\Upsilon(2 s)$ & 4.32 & 3.8 & 3.70 & 3.9 & 2.05 & $1.3 \pm 0.85$ \\
$\Upsilon(3 s)$ & 2.20 & 2.0 & 1.92 & 2.07 & 1.05 & $<3.4$ \\
\hline \hline
\end{tabular}



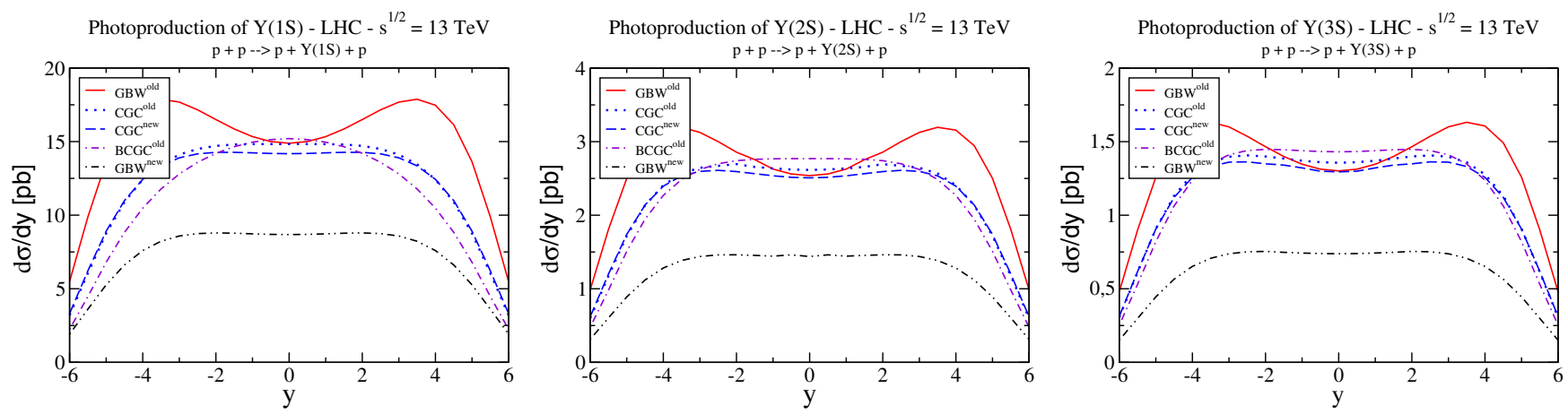

FIG. 2. Rapidity distribution for the vector meson states $\Upsilon(1 S)$ (left panel), $\Upsilon(2 s)$ (central panel), and $\Upsilon(3 S)$ (right panel) in $p p$ collisions in the LHC Run II at $\sqrt{s}=13 \mathrm{TeV}$. The same notation as in the previous figure is used.

predicted at lower energy, including the theoretical uncertainty and overall behavior. The cross sections are quite sizeable, and the relative contribution of the radial excited states compared to the lowest state follows the pattern shown at $y=0$. Namely, $\sigma[\Upsilon(2 S)] / \sigma[\Upsilon(1 S)] \simeq$ 0.17 , and $\sigma[\Upsilon(3 S)] / \sigma[\Upsilon(1 S)] \simeq 0.083$. There is a discrepancy at forward rapidities when GBW old is compared to the other models. The reason could be the typically higher saturation scale associated to that model $\left(Q_{\text {sat }}^{2}=1 \mathrm{GeV}^{2}\right.$ at $x=4 \times 10^{-5}$ for GBW old, whereas it reaches one at $x=5.5 \times 10^{-7}$ for GBW-KSX). In Table IV, the integrated cross sections are shown for the states $\Upsilon(1 S, 2 S, 3 S)$ and $\psi(1 S, 2 S)$ corrected for acceptance. Typically, the cross sections are $40 \%$ higher compared the LHC Run I at $7 \mathrm{TeV}$. There is a difference among the shapes of the distributions obtained from the dipole cross sections considered. One could therefore expect that measurements of the rapidity distributions would be able to discriminate between models. Our predictions for the $\Upsilon$ state ratios are lower than those predicted by STARlight Monte Carlo, as presented in Ref. [15]. The origin can be the fact that the different states are obtained from an extrapolation of HERA-DATA and using a fixed ratio for the distinct states in Ref. [15]. In our case, the evolution on energy is dynamically generated by parton saturation approach models, and the meson wave funtions have nontrivial behavior on the overlap function.

We now turn to the prediction in $p A$ ultraperipheral collisions. In particular, in proton-lead collisions, if the quarkonium rapidity, $y$, is positive in the nucleus beam direction, its rapidity distribution reads as [1]

TABLE IV. Predictions for integrated cross sections (in units of $p b)$ for photoproduction of the $\psi(1 S, 2 S)$ and $\Upsilon(1 S, 2 S, 3 S)$ states in $p p$ collisions at the LHC Run II $(\sqrt{s}=13 \mathrm{TeV})$.

\begin{tabular}{lrcccc}
\hline \hline & GBW & CGC $^{\text {old }}$ & CGC $^{\text {new }}$ & BCGC $^{\text {old }}$ & GBW $^{k s x}$ \\
\hline$\psi(1 s)$ & 997.52 & 747.75 & 696.25 & 523.3 & 598.96 \\
$\psi(2 s)$ & 31.92 & 21.9 & 22.02 & 14.52 & 16.15 \\
$\Upsilon(1 s)$ & 43.77 & 34.3 & 33.8 & 30.97 & 20.6 \\
$\Upsilon(2 s)$ & 7.72 & 6.5 & 6.37 & 6.45 & 3.45 \\
$\Upsilon(3 s)$ & 3.95 & 3.42 & 3.35 & 3.47 & 1.77 \\
\hline \hline
\end{tabular}

$$
\begin{aligned}
& \frac{d \sigma}{d y}(P b+p \rightarrow P b+p+V) \\
& \quad=\frac{d N_{\gamma}^{P b}(y)}{d \omega} \sigma_{\gamma p \rightarrow V+p}(y)+\frac{d N_{\gamma}^{p}(-y)}{d \omega} \sigma_{\gamma P b \rightarrow V+P b}(-y),
\end{aligned}
$$

where $\frac{d N_{\gamma}(y)}{d \omega}$ is the corresponding photon flux. The case for the inverse beam direction is straightforward. We use the Weiszäcker-Williams method to calculate the flux of photons from a charge $Z$ nucleus as referred to in the previous section. In the numerical calculation, we disregard the contribution coming from the photon flux related to the proton source. For comparison, concerning the $\psi(1 S)$ and $\psi(2 S)$ states at $\sqrt{s}=5.02 \mathrm{TeV}$, the integrated cross section (in the range $2 \leq y \leq 4.5$ ) is predicted to be $243.41 \pm 20.37$ and $4.97 \pm 0.48 \mathrm{pb}$, respectively. The error includes the theoretical uncertainty related to the model of the dipole cross section. When considering the higher energy of $\sqrt{s}=8.2 \mathrm{TeV}$, the values found are $340.51 \pm$ 36.76 and $7.14 \pm 0.85 \mathrm{pb}$. Moreover, for the lowest state $\Upsilon(1 S)$, one has $2.92 \pm 1.43 \mathrm{pb}$ at $\sqrt{s}=5.02 \mathrm{TeV}$ and $5.45 \pm 2.04 \mathrm{pb}$ at $\sqrt{s}=8.2 \mathrm{TeV}$.

In Fig. 3, the rapidity distribution is shown for the three $\Upsilon$ states in $p A$ collisions at the energy $\sqrt{s}=8.2 \mathrm{TeV}$. The model deviation is more intense on the very forward rapidity and the theoretical uncertainty reaches a factor 2 similarly to the $p p$ case. The rapidity distribution exhibits directly the influence of the $x$ dependence of the dipole cross section in the interval $0 \leq y \leq 4$ and especially for rapidities around $y=3$. The shapes are similar for the lowest state and its radial excited states. Therefore, it is feasible that a consideration of $\Upsilon$ photoproduction in this rapidity interval offers potential in discriminating models of dipole cross sections. It is timely having a measurement of $\Upsilon$ production at midrapidity, as the corresponding cross section in the $\psi$ case is a challenge when considering the color dipole approaches. The predictions can be directly compared to the work in Ref. [33], where the perturbative two-gluon exchange formalism has been considered. We have not verified a second peak in the rapidity distribution as presented in Ref. [33]. The reason is that the dynamics 

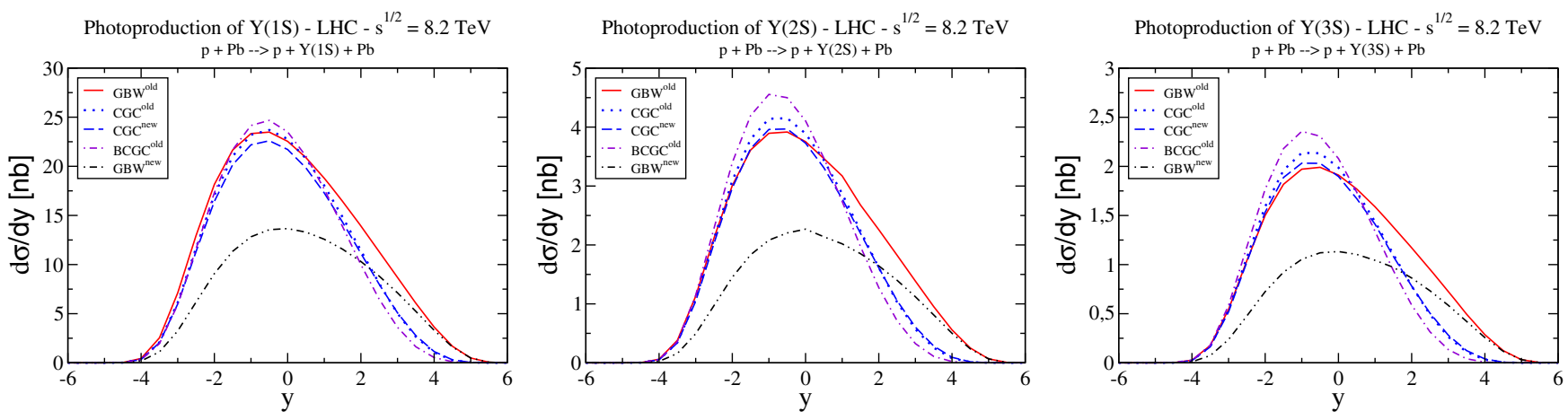

FIG. 3. Rapidity distribution for the vector meson states $\Upsilon(1 S)$ (left panel), $\Upsilon(2)$ (central panel), and $\Upsilon(3 S)$ (right panel) in $p A$ collisions at $\sqrt{s}=8.2 \mathrm{TeV}$.

embedded in the color dipole approach considered here corresponds to strong shadowing corrections. The ratios $\sigma[\Upsilon(2 S)] / \sigma[\Upsilon(1 S)]$ and $\sigma[\Upsilon(3 S)] / \sigma[\Upsilon(1 S)]$ are still of the same order of magnitude compared to the protonproton case.

Finally, let us consider the $A A$ collisions. For coherent $A A$ ultraperipheral collisions, the rapidity distribution of vector meson $V$ is given by

$$
\begin{aligned}
\frac{d \sigma}{d y}(A+A \rightarrow A+A+V)= & {\left[\omega \frac{d N_{\gamma}^{P b}(\omega)}{d \omega} \sigma(\gamma A \rightarrow V+A)\right.} \\
& +(y \rightarrow-y)] .
\end{aligned}
$$

Figure 4 shows the rapidity distribution for the $\Upsilon$ states at the energy $\sqrt{s}=5.5 \mathrm{TeV}$ (the notation is the same as in previous figures). The distributions are symmetric about midrapidity and also similar in structure, and the influence of distinct models for dipole cross section is significantly less evident than in the $p A$ case. Cross sections are still sizeable, and the relative contribution of radial excited states compared to $\Upsilon(1 S)$ is given by $\sigma[\Upsilon(2 S)] / \sigma[\Upsilon(1 S)] \simeq$ 0.15 and $\sigma[\Upsilon(3 S)] / \sigma[\Upsilon(1 S)] \simeq 0.075$, which is slightly smaller than in the $p p$ case. Now, the midrapidity region seems to show sensitivity to discriminate models of dipole cross section and corresponding nuclear effects. Compared to the STARlight Monte Carlo [15], now our predictions are quite similar for the $\Upsilon(1 S)$ state (we show the large theoretical uncertainty in modeling the dipole cross sections) but lower values for the $\Upsilon(2 S, 3 S)$ states. In general, the Glauber model considered in Ref. [15] involves less nuclear shadowing than the Glauber-Gribov approach we have used. In Ref. [33], the $\Upsilon$ production also was analyzed in $A A$ collisions, and we verify that the theoretical uncertainty in the color dipole approach is comparable to that present in perturbative two-gluon exchange formalism. In present study only the coherent channel is considered. An important investigation would be the inclusion of ultraperipheral collisions accompanied by photonuclear breakup. The procedure for obtaining this involves the multiplication of the original photon flux by a probability factor for the desired nuclear breakup.

The formulation to obtain the transverse-momentum distribution for the exclusive photoproduction of $\Upsilon$ states was also explored. Figure 5 presents some samples of results concerning the $1 S$ state. The left panel shows the prediction for $p p$ collisions in the energy value of the LHC Run II, $\sqrt{s}=13 \mathrm{TeV}$. In addition, the right panel presents the same distribution in $A A$ collisions at $\sqrt{s}=5.5 \mathrm{TeV}$. For the $p p$ case, the Gaussian behavior is natural as we are imposing an exponential behavior for the $t$ dependence which is ad hoc and consistent with the experimental results from DESYHERA on vector meson photoproduction. On the other
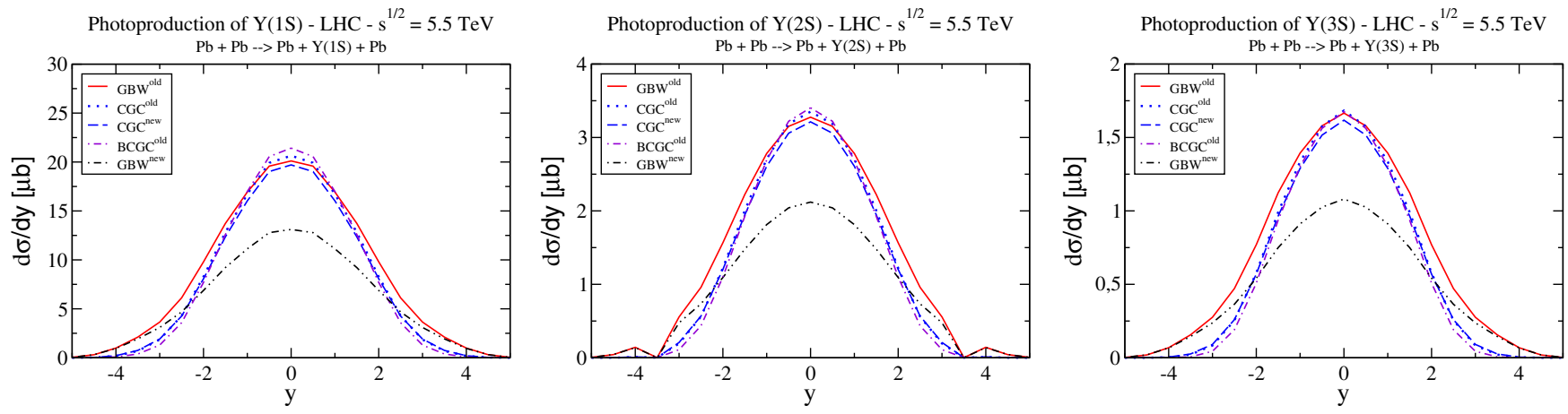

FIG. 4. Rapidity distribution for the $\Upsilon(1 S, 2 S, 3 S)$ states in PbPb collisions at $\sqrt{s}=5.5 \mathrm{TeV}$. 

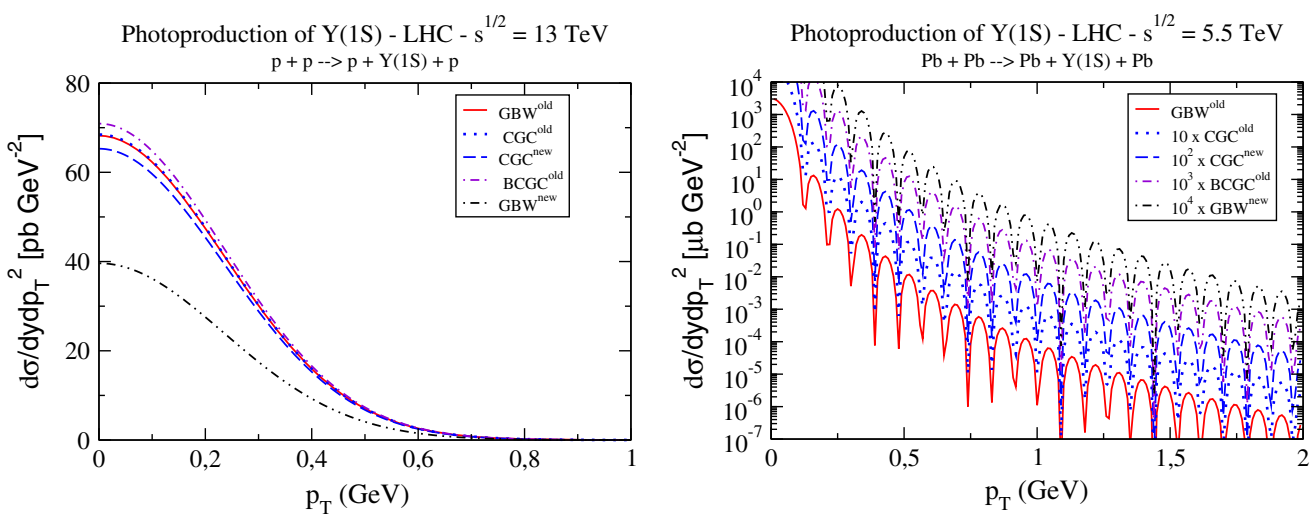

FIG. 5. Transverse momentum distribution for the $\Upsilon(1 S)$ state in $p p$ collisions at $\sqrt{s}=13 \mathrm{TeV}$ (left panel) and $P b P b$ collisions at $\sqrt{s}=5.5 \mathrm{TeV}$ (right panel).

hand, for the $A A$ case, the rich structure comes from the impact parameter dependence of the nuclear dipole amplitude. Concerning the $p_{T}$ behavior in $p p$ collisions, it was discussed in Ref. [20] that proton detectors and cuts on meson transverse momentum facilitate a measurement of the $\gamma p \rightarrow \Upsilon p$ cross section at $W_{\gamma p} \approx 1 \mathrm{TeV}$, which corresponds to the range in which saturation effects are expected to be revealed.

\section{SUMMARY}

We have considered exclusive photoproduction of $\Upsilon$ states in ultraperipheral $p p, p P b$, and $P b P b$ collisions at the LHC, using the color dipole approach as the underlying theoretical framework. The rapidity and transverse- momentum distributions, integrated cross section, and cross section ratios for these collisions have been presented and compared with available data. The significative dependence on the dipole cross section could be useful in discriminating models, mostly in the proton-nucleus case at forward rapidities. Our main goal is to provide predictions taking into account a theoretical framework which correctly describes the radially excited states.

\section{ACKNOWLEDGMENTS}

This work was partially financed by the Brazilian funding agency $\mathrm{CNPq}$ and Rio Grande do Sul funding agency FAPERGS.
[1] G. Baur, K. Hencken, D. Trautmann, S. Sadovsky, and Y. Kharlov, Phys. Rep. 364, 359 (2002); C. A. Bertulani, S. R. Klein, and J. Nystrand, Annu. Rev. Nucl. Part. Sci. 55, 271 (2005).

[2] N. N. Nikolaev and B. G. Zakharov, Phys. Lett. B 332, 184 (1994); Z. Phys. C 64, 631 (1994).

[3] J. Nemchik, N. N. Nikolaev, E. Predazzi, and B. G. Zakharov, Phys. Lett. B 374, 199 (1996).

[4] M. B. Gay Ducati, M. T. Griep, and M. V. T. Machado, Phys. Rev. D 88, 017504 (2013).

[5] M. B. Gay Ducati, M. T. Griep, and M. V. T. Machado, Phys. Rev. C 88, 014910 (2013).

[6] R. Fiore, L. Jenkovszky, V. Libov, and M. V. T. Machado, Theor. Math. Phys. 182, 141 (2015); Teor. Mat. Fiz. 182, 171 (2015); arXiv:1408.0530.

[7] R. Fiore, L. Jenkovszky, V. Libov, M. V. T. Machado, and A. Salii, AIP Conf. Proc. 1654, 090002 (2015); arXiv:1506.01990.

[8] B. Abelev et al. (ALICE Collaboration), Phys. Lett. B 718, 1273 (2013).
[9] E. Abbas et al. (ALICE Collaboration), Eur. Phys. J. C 73, 2617 (2013).

[10] R. Aaij et al. (LHCb Collaboration), J. Phys. G 40, 045001 (2013).

[11] R. Aaij et al. (LHCb Collaboration), J. Phys. G 41, 055002 (2014).

[12] F. Gelis, E. Iancu, J. Jalilian-Marian, and R. Venugopalan, Annu. Rev. Nucl. Part. Sci. 60, 463 (2010); H. Weigert, Prog. Part. Nucl. Phys. 55, 461 (2005); J. Jalilian-Marian and Y. V. Kovchegov, Prog. Part. Nucl. Phys. 56, 104 (2006).

[13] M. B. Gay Ducati, Braz. J. Phys. 31, 115 (2001); A. Ayala, M. B. Gay Ducati, and E. M. Levin, Eur. Phys. J. C 8, 115 (1999); Phys. Lett. B 388, 188 (1996); Nucl. Phys. B493, 305 (1997); A. Ayala, M. B. Gay Ducati, and E. M. Levin, Nucl. Phys. B511, 355 (1998).

[14] R. Aaij et al. (LHCb Collaboration), J. High Energy Phys. 09 (2015) 084.

[15] S. R. Klein, J. Nystrand, J. Seger, Y. Gorbunov, and J. Butterworth, arXiv:1607.03838. 
[16] S. Klein and J. Nystrand, Phys. Rev. C 60, 014903 (1999).

[17] R. Chudasama et al. (CMS Collaboration), arXiv:1607.00786.

[18] G. S. Santos and M. V. T. Machado, J. Phys. G 42, 105001 (2015).

[19] J. Nemchik, N. N. Nikolaev, E. Predazzi, and B. G. Zakharov, Z. Phys. C 75, 71 (1997).

[20] B. E. Cox, J. R. Forshaw, and R. Sandapen, J. High Energy Phys. 06 (2009) 034.

[21] K. Golec-Biernat and M. Wüsthoff, Phys. Rev. D 59, 014017 (1998).

[22] M. Kozlov, A. Shoshi, and W. Xiang, J. High Energy Phys. 10 (2007) 020.

[23] E. Iancu, K. Itakura, and S. Munier, Phys. Lett. B 590, 199 (2004).

[24] H. Kowalski, L. Motyka, and G. Watt, Phys. Rev. D 74, 074016 (2006).

[25] I. Balitsky, Nucl. Phys. B463, 99 (1996).

[26] Y. V. Kovchegov, Phys. Rev. D 60, 034008 (1999).

[27] Y. V. Kovchegov, Phys. Rev. D 61, 074018 (2000).

[28] G. Soyez, Phys. Lett. B 655, 32 (2007).
[29] A. H. Rezaeain and I. Schmidt, Phys. Rev. D 88, 074016 (2013).

[30] W. Schäfer and A. Szczurek, Phys. Rev. D 76, 094014 (2007); A. Rybarska, W. Schäfer, and A. Szczurek, Phys. Lett. B 668, 126 (2008).

[31] S. P. Jones, A. D. Martin, M. G. Ryskin, and T. Teubner, J. Phys. G 43, 035002 (2016).

[32] V. P. Gonçalves, B. D. Moreira, and F. S. Navarra, Phys. Lett. B 742, 172 (2015); V. P. Gonçalves, B. D. Moreira, F. S. Navarra, and D. Spiering, Phys. Rev. D 94, 014009 (2016); G. Gil da Silveira, V. P. Gonçalves, and M. M. Jaime, arXiv:1609.09854.

[33] A. Adeluyi and A. Nguyen, Phys. Rev. C 87, 027901 (2013).

[34] C. Adloff et al. (H1 Collaboration), Phys. Lett. B 541, 251 (2002).

[35] J. Bartels, K. J. Golec-Biernat, and K. Peters, Acta Phys. Pol. B 34, 3051 (2003).

[36] G. P. Lepage and S. J. Brodsky, Phys. Rev. D 22, 2157 (1980). 